\title{
Le vécu de la sexualité chez les paraplégiques
}

\author{
Eric J.J. BERARD
}

Centre Médical de l'Argentì̀re, 69610 Aveize

\section{RESUME}

La paraplégie modifie les réponses sexuelles de l'homme. Au dessus de T10, les réactions sont de type réflexe non ressenties et des artifices érotiques sont nécessaires.

Dans les atteintes basses complètes, des artifices techniques doivent pallier l'impuissance et l'anéjaculation mais ceux-ci sont souvent mal tolérés.

Dans les atteintes D12 L1 incomplètes, les réactions dont le caractère reste incomplet ne se font qu'en réponse à un mécanisme psychogène.

L'ajustement de chaque paraplégique se fait dans le cadre d'un travail de deuil qu'il faut bien connaître pour pouvoir l'accompagner sur le plan médico-psychologique en laissant du temps au temps.

Mots clés : Paraplégie, Sexualité, Deuil.

\section{INTRODUCTION}

Dans leur dimension universelle, l'homme et la femme répondent à des critères génétiques, anatomiques et psycho-physiologiques communs, parmi lesquelles les réponses génito-sexuelles [11].

Bien que le vécu génito-sexuel des hommes paraplégiques soit aussi marqué par cette dimension universelle qui nous caractérise tous, les paraplégiques ont, eux, des particularités qu'il convient de connaitre, au même titre que l'histoire, unique, propre à chacun, si on veut prendre en compte toutes les dimensions de leur vécu génito-sexuel. STEnsman, en 1989, dans un travail sur l'image du corps, montre en effet que la réaction de chacun vis à vis de cette image procède d'une expérience individuelle qu'aucun schéma standard ne peut généraliser [15].

\section{LES REACTIONS SEXUELLES DANS LES DIFFERENTS SYNDROMES NEUROLOGIQUES}

Les réponses sont particulières à chaque catégorie de syndromes, chez l'homme comme chez la femme.

1. Dans les syndromes complets au dessus de T10, bien que la libido ne soit pas directement affectée par la lésion médullaire, les réponses génito-sexuelles aux stimulations sont quant à elles de caractère purement réflexe, non maitrisées par le sujet et ne donnant pas lieu à une sensation directe de plaisir. Dès lors, des artifices érotiques peuvent permettre à l'homme, lorsqu'il est paraplégique, de partager un plaisir physique avec l'autre, ce qui nécessite certaines positions particulières permettant de stimuler les zones érogènes sus-lésionnelles [10]. L'intégration de ces stimulations à des fantasmes permet en effet de retrouver un nouveau type d'orgasme.

Quand le niveau de la lésion médullaire est haut, notamment cervical, ces sensations para-orgasmiques peuvent être vécues de 
façon désagréable, voire insupportable, sous forme de réactions d'hyper-réflectivité autonome. On rencontre également souvent ces réponses quand on pratique un vibromassage pour obtenir une éjaculation chez l'homme tétraplégique.

L'homme ne commande qu'exceptionnellement son éjaculation au cours de son rapport. A des niveaux hauts d'atteinte médullaire (de C4 à D5), l'éjaculation peut être par contre obtenue par auto ou hétéro-masturbation mais le vibro-massage peut être nécessaire, associé même à une injection d'ESERINE pour faciliter l'éjaculation antérograde. Ces techniques, outre le fait qu'elles nécessitent une médiation médicale, sont le plus souvent mal vécues, et par le patient qui présente régulièrement un malaise physique, et par la conjointe quand elle participe à l'"opération“.

2. Quand au moins une partie des centres sympathiques dorso-lombaires centrés sur T12-L1 est intacte, l'homme paraplégique peut avoir des réactions psycho-physiologiques perçues comme ressemblant aux réponses normales avec une érection ébauchée, dont le mécanisme est psychogène, mais où l'orgasme est très atténué ou absent, ce qui entraine souvent une sensation de frustration pour le blessé médullaire. Chez ce dernier, cependant, l'émission de sperme existe, sans phase d'expulsion rythmée, ce qui n'autorise pas à employer le terme d"éjaculation psychogène" dans ces cas, mais plutôt d'émission du sperme d'origine psychogène.

3. Quand les centres nerveux dorsolombaires et sacrés sont détruits, de même que dans les syndromes de la queue de cheval, il n'y a plus aucune réponse psycho-physiologique en relation avec la sphère génito-sexuelle : l'homme est impuissant. La femme paraplégique ne consulte pas pour elle, car, ne ressentant pas, elle est moins gênée par son état que le partenaire, chez lequel peut par contre se développer une impuissance psychogène réactionnelle.
Quant à l'homme paraplégique impuissant, il consulte, lui par contre, dans le but de restaurer et sa virilité, et sa fertilité. Parmi les artifices techniques qu'on peut lui proposer vis à vis de l'impuissance, l'injection intra-caverneuse de vaso-dilatateurs est d'autant plus angoissante pour lui qu'on doit l'informer préalablement des risques non négligeables d'hématome local et surtout de priapisme. Quant aux prothèses péniennes, elles ne sont proposées qu'après un certain temps, et après avoir essayé d'autres solutions. Ces prothèses induisent parfois un syndrôme névrotique chez le paraplégique, mais aussi chez sa conjointe, pouvant conduire à la séparation du couple.

Devant le syndrôme anéjaculatoire, on ne peut, à ces niveaux d'atteinte neurologique bas que proposer une technique d'électroéjaculation, au moyen d'une sonde intrarectale [9]. En dehors du fait qu'on n'obtient pas toujours du sperme, qu'il n'est pas toujours d'une bonne qualité fécondante, et que cela est très frustrant, l'homme paraplégique et son épouse [1] gardent en général un mauvais souvenir de cette expérience, comme d'un geste déshumanisant.

\section{IDENTITE SEXUELLE ET VECU DE DEUIL}

Le deuil est une expérience unique, propre à chacun, qui comporte chez le paraplégique des caractéristiques particulières et un rapport direct avec le vécu génito-sexuel $[5,6]$.

En 1988, nous avions fait réaliser à certains paraplégiques leur auto-portrait [4]. Ce test révéla une image atrophiée du corps, à laquelle se substituait temporairement une représentation mentale symbolique. Les entretiens de ces mêmes paraplégiques avec le psychologue faisaient ressortir une perte de l'image valorisée de soi, d'autant plus forte que la personnalité était antérieurement plus narcissique.

Dans les entretiens avec les patients les plus avancés dans leur processus d'ajustement manifestaient un intérêt pour leur 
fonctionnement génito-sexuel, tandis que les autres manifestaient un déni ou un blocage accompagnant des signes d'angoisse. Les variations dans les réactions sont aussi fonction du caractère neurologique de la lésion médullaire appréciée selon la classification de FrANKEL.

De l'étude du contenu onirique de ces paraplégiques, il ressortait, cas par cas, un éclairage du processus inconscient du deuil, mais aucune généralisation ne doit être faite à partir de cette "grille de lecture", qui ne peut que rester individuelle.

Des groupes de parole avec les familles de ces paraplégiques ont mis en évidence le décalage des processus d'ajustement à la paraplégie entre le patient d'une part et le conjoint ou les parents d'autre part.

Dans le cadre de la même étude, un travail en dynamique de groupe avec les soignants a montré la peur de ceux-ci de s'investir dans la relation avec le patient et la négation de son identité sexuelle, la nudité étant minimisée dans son importance (à la différence de ce qu'expriment les soignants anglo-saxons). Enfin, certains soignants nient toute dimension sexuelle à leur relation privilégiée avec certains patients.

\section{EVOLUTION CHRONOLOGIQUE DU VECU SEXUEL}

\section{Dans les premières semaines suivant l'accident}

Le blessé, installé dans une dépendance complète, est sous le coup du traumatisme physique et psychique. Son corps est altéré et son identité antérieure est globalement remise en question. Le corps, morcelé et insensible, est l'objet des soins, où, dans la nudité souvent découverte et banalisée, le sexe perd de sa valeur symbolique. Une modification aussi forte de son corps malade et de son sexe ne peut pas ne pas entraîner des réactions d'inquiétude profonde, voire d'angoisse, et, du coup, de régression psychique. On comprend alors l'importance d'un cadre déontologique et éthique qui puisse servir de référence à chacun pour se situer face à des réactions parfois peu rationnelles.

L'homme paraplégique subit une altération de son image [8] pouvant entre autres réveiller le fantasme de castration, que la paraplégie soit flasque et qu'il perde alors les signes visibles de sa virilité ou que sa paraplégie revête au contraire des caractères de réflectivité, l'érection posant alors le problème d'être déclenchée par des gestes soignants extérieurs à lui-même. Une telle situation donne souvent lieu à des ambiguités relationnelles et peut nourrir des fantasmes, autant chez le soigné que chez le ou la soignante.

Le langage passant par le corps (frictions, massages, toilette), fait partie intégrante des relations du type "mère- nourrisson" correspondant bien à la partie de la sexualité du blessé médullaire régressée à une phase archaĩque [2]. Le vécu du corps à cette phase est très fort : "je me suis senti comme coupé en deux ${ }^{4}$, nous a confié un de nos paraplégiques.

\section{Le réinvestissement du corps}

Après la phase initiale de dépendance, le blessé médullaire fait l'expérience de son auto-prise en charge ; dans ce cadre, la technique de l'auto-sondage facilite le retour du sujet à un stade d'auto-sexualité, où la redécouverte des organes génitaux rappelle la période de l'adolescence. Phase d'épanouissement narcissique, elle s'accompagne de manoeuvres de séduction ; phase d'auto-exploration, elle confronte le blessé médullaire aux tabous de la masturbation.

Toujours intriquée avec la sexualité, l'agressivité se manifeste très tôt chez le blessé médullaire. Elle accompagne constamment un état de détresse et de carence psychoaffective. A l'origine de cette agressivité existe souvent une tentative de séduction qui a échoué ou au contraire trop bien réussi, entrainant de la part du soignant un comportement qui appelle la défense du patient sous forme aggressive. 
Le vécu érotique est, avec les fonctions relationnelle et procréatrice, une composante maîtresse de l'équilibre sexual permettant d'investir son corps comme source de plaisir [16]. Or, le blessé médullaire ne ressent plus son corps paralysé, ce qui l'amène à rechercher des manifestations différentes du plaisir antérieurement éprouvé. Si le blessé médullaire accepte de s'ouvrir à l'altérité de son image corporelle, le réinvestissement de son corps altéré peut lui conférer une image valorisante et participer à la reconstitution de son identité sexuelle.

\section{La démarche hétéro-sexuelle du blessé médullaire}

Après la phase précédente d'auto-sexualité, mûrie dans le réinvestissement du corps où la fonction érotique était au premier plan, le blessé médullaire cherche à retrouver son identité sexuelle en fonction de l'autre, en fonction de son vécu antérieur et de ses conditions de vie actuelles. La notion de couple transparait dans les discussions avec l'équipe soignante et avec le médecin, les angoisses et les difficultés étant exprimées en premier. Celles-ci sont apaisées si, à partir de cette écoute, on reprend avec chaque blessé médullaire ses possibilités particulières en expliquant les réactions psychophysiologiques qui correspondent à son état d'homme spinal. Dans cet esprit, les nouvelles réponses génito-sexuelles possibles sont envisagées, ainsi que les sollicitations susceptibles de les faciliter : verbalisation, stimulations préliminaires ... [12, 14] Une intégration des deux hémi-corps est possible dans toutes les hypothèses et elle permet une relation avec l'autre, partagée dans le plaisir. L'élément relationnel et l'érotisme peuvent ainsi se rééquilibrer plus harmonieusement.

\section{Face au couple}

Dans la perspective de l'utilisation de techniques de stimulation de l'érection et de l'éjaculation, le médecin doit se poser plusieurs questions : a) la médiation objectale technique n'estelle pas perturbante pour l'épanouissement de l'amour dans le couple?

b) comment doit-on faire intervenir la partenaire dans l'utilisation de ces techniques?

c) comment juger des possibilités de compensation homéostasiques des partenaires face à la médicalisation?

d) jusqu'où l'intervention médicale peut-elle s'impliquer dans la relation du couple?

e) quelle est la signification du désir d'enfant?

Ces questions doivent être abordées au niveau du couple, en les resituant dans la trilogie relationnel-érotique-procréatif.

A côté des questions que doit se poser tout médecin confronté à un couple où l'homme est paraplégique, il est des questions des patients eux-mêmes ou de leur conjointe qu'il faut savoir entendre ou même solliciter. Il faut admettre que la relation-plaisir appartient au couple lui-même mais qu'il appartient au médecin de répondre aux demandes d'information sur les zones érogènes et les moyens de déclencher les réactions psychosexuelles.

Il appartient également au médecin de répondre à l'angoisse de la flaccidité quand elle existe et de laisser entrevoir son caractère éventuellement régressif avec le temps.

Cette question de temps joue un grand rôle dans l'ajustement et la résolution du vécu de deuil, on peut aussi se poser la question de la façon de présenter les artifices techniques palliatifs de l'érection. Le risque est en effet de les faire passer avant l'ajustement psychologique de l'homme paraplégique. De même pour la fertilité, la mise en avant des techniques de stimulation n'estelle pas qu'apparemment rassurante sur l'avenir de la fonction de procréation ?

Pendant le temps de plus en plus court de la période de rééducation en centre où a lieu la gestion de ces questions avec le blessé 
médullaire et éventuellement son conjoint, on peut avoir atteint la fin du séjour, sans que l'identité sexuelle n'ait été retrouvée. Il faut alors savoir donner du temps au temps et se tenir à la disposition du blessé médullaire et de son conjoint dans le cadre du service de suite pour l'aider à s'ajuster à son handicap [6].

\section{REFERENCES}

1. ALNOT M.O. : Les femmes face à la procréation assistée : perspective et santé. In : La santé au féminin. $1986 ; 39: 27-30$.

2. BERARD E., FYON J.P., TALLENT A. et al. : Les paraplégiques sont-ils égaux devant l'escarre ? In : Les escarres. Sous la direction de L. SIMON et J. PELISIER. Masson 1984 ; 38-47.

3. BERARD E., BOUCAND M.H., THEVENOT X. : Le médecin rééducateur confronté à la sexualité des blessés médullaires : repères éthiques. In : Actualités en rééducation. Sous la direction de $L$. SIMON et J. PELISSIER. Masson $1988 ; 102$ 109.

4. BERARD E., BOUCAND M.H., COZZANI E. et al. : The mourning process in spinal cord injury. In : Proc. 29th annual scientific meeting IMSOP. ROMA. 1989.

5. BERARD E. \& BOUCAND M.H. : Annonce du handicap et identité sexuelle du blessé médullaire. In : Actes du congrès AFIGAP. Lyon. Hospices Civils Lyon Ed. $1990 ; 1-8$.

6. BOUCAND M.H., BERARD E., BOTTA J.M. et al. : Deuil et handicap. In : Ann. Réad. \& Med. Phys. $1989 ; 3 ; 277-283$.

7. FARRE J.M. : Sexualidad humana : en que consiste ? In : Sexualidad y lesion medular. M.L. CURCOLL \& J. VIDAL. Fundacio institut Guttman Ed. Barcelona 1992 ; 17-25.

8. FRANCOIS N. \& MAURY M. : Sexual aspects in paraplegic patients. In : Paraplegia $1987 ; 25 ; 289$ 292.

9. HALSTEAD L.S., VERVOORT S. \& SEAGER S.W. : Rectal probe electrostimulation in the treatment of anejaculatory spinal cord injured men. In : Paraplegia $1987 ; 25 ; 120-129$.

10. LERICHE A. \& MINAIRE P. : La rééducation sexuelle du blessé médullaire de sexe masculin. In : La paraplégie. Sous la direction de P. MINAIRE. Masson 1982 ; 199-204.

11. MASTERS W. \& JOHNSON V. : Human sexual response. In : Little brown. Boston 1965.
12. NIKAS V., FLEISCHMAN P. \& BURTON P. Sexual and marital adjustment of World War II spinal cord injured veterans. In : Paraplegia 1990 ; 28 ; 164-171.

13. PARRINDER G. : Le sexe dans les religions du monde. In : Le Centurion. Mulhouse 1983.

14. SIOSTEEN A. \& al. : Sexual ability, activity, attitudes and satisfaction as part of adjustment in spinal cord injured subjects. In : Paraplegia $1990 ; 28$; 285-295.

15. STENSMAN R. : Body image among 22 persons with acquired \& congenital impairement. In : Paraplegia $1989 ; 27 ; 27-36$.

16. THEVENOT X. : Repères éthiques pour un monde nouveau. In : 3ème Ed. Salvator. Mulhouse 1983.

\section{ABSTRACT \\ How paraplegic patients live their sexuality}

\author{
J.J. BERARD
}

Centre Médical de l'Argentière, 69610 Aveize

In spinal cord injury, sexual reactions have a common dimension with ablebodied mankind, but also particular features corresponding to particular neurological syndromes. The third dimension which has to be taken into account is the singular one, every man, paraplegic or able-bodied, being unique.

When the level of the lesion is above T10, genito-sexual responses to stimulations have a reflex functioning. In such cases, erotic tricks must be used to complete psycho-physiological reactions. On the contrary, technical devices are ill-tolerated both by the paraplegic man and by his spouse.

Incomplete lesions in T12-L1 have to psychogenic responses to stimulations, i.e. partial erection and/or semen emission due to the neurophysiological mecanisms of desire.

In the complete lesions of the conus medullaris or of the cauda equina, men become impotent and can no longer produce semen. In such cases, techni- 
cal devices and drugs directly injected in the corpus cavernosum are technically efficacious but psychologically ill-tolerated.

Facing his new sexual responses, the paraplegic man and his spouse have to adjuste themselves to the new circumstances. The way of adjustment is similar to a mourning process.
One has to pay attention to let every paraplegic live every period of adjustment on his own, and at the same time one must be present to help him and answer his questioning.

Key words : spinal cord injury - sexuality mourning process 